This document is confidential and is proprietary to the American Chemical Society and its authors. Do not copy or disclose without written permission. If you have received this item in error, notify the sender and delete all copies.

\title{
Remotely controlling the crystallization of thin polymer coatings
}

\begin{tabular}{|r|l|}
\hline Journal: & Macromolecules \\
\hline Manuscript ID & ma-2020-008872.R2 \\
\hline Manuscript Type: & Article \\
\hline Author: & $20-$ May-2020 \\
\hline Complete List of Authors: & $\begin{array}{l}\text { Vanroy, Bram; KU Leuven } \\
\text { Wübbenhorst, Michael; K.U.Leuven, Department of Physics and } \\
\text { Astronomy } \\
\text { Napolitano, Simone; Universite Libre de Bruxelles, Department of } \\
\text { Physics }\end{array}$ \\
\hline
\end{tabular}

\section{SCHOLARONE \\ Manuscripts}




\title{
Remotely controlling the crystallization of thin polymer coatings
}

\author{
Bram Vanroy ${ }^{1}$, Michael Wübbenhorst ${ }^{1}$, Simone Napolitano ${ }^{2, *}$ \\ ${ }^{1}$ Laboratory for Soft Matter and Biophysics, Department of Physics and Astronomy, KU Leuven, \\ Celestijnenlaan 200D, Leuven, 3001, Belgium \\ ${ }^{2}$ Laboratory of Polymer and Soft Matter Dynamics, Faculté des Sciences, Université libre de Bruxelles \\ (ULB), Boulevard du Triomphe, Brussels 1050, Belgium \\ emailsnapolit@ulb.ac.be
}

\section{For Table of Contents Use Only}

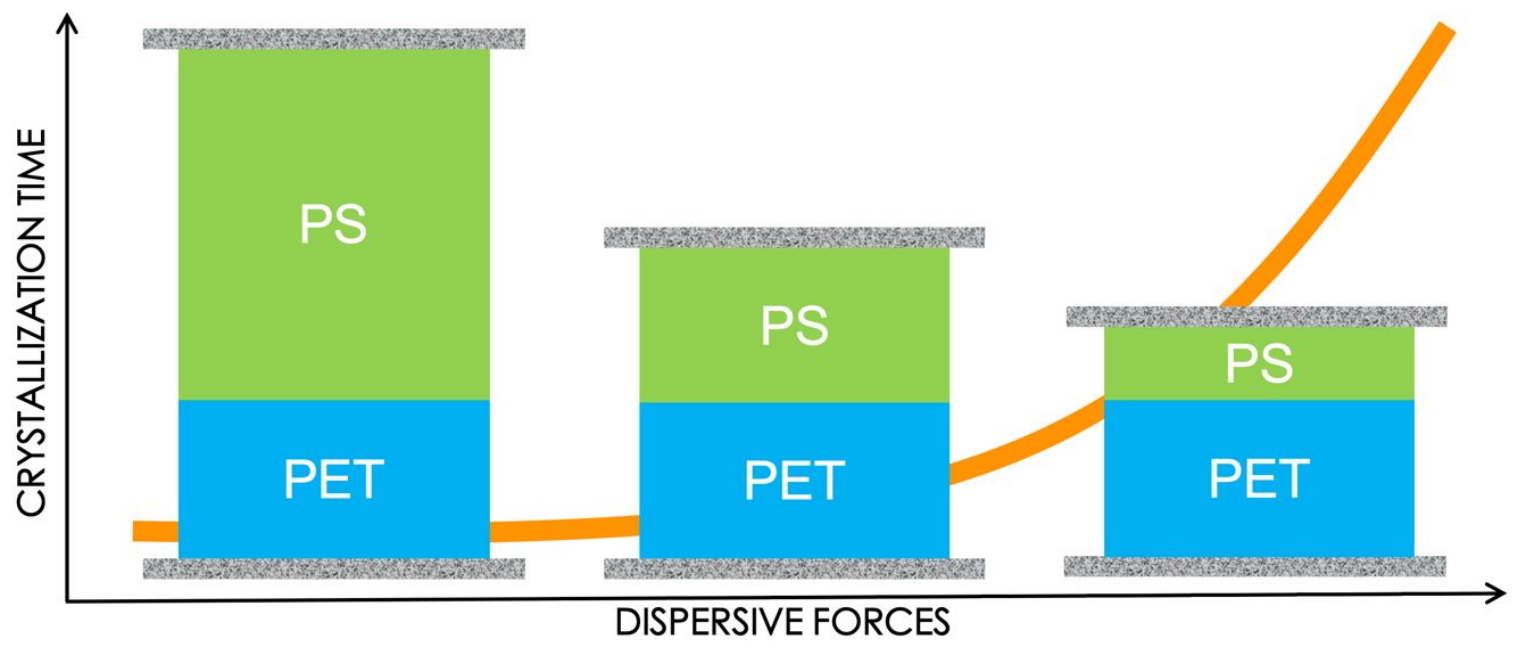




\begin{abstract}
We introduce a method permitting remote control of the crystallization of thin polymer films, not requiring changes in the film thickness or the interfacial chemistry. The procedure is based on the assembly of hybrid multilayers, where ultrathin films of a crystallizable polymer poly(ethylene terephthalate), PET - are embedded in parallel with noncrystalline polymer layers - polystyrene, PS - into metallic slabs. Variation of the size of the PS film allowed tuning the crystallization rate of PET layers of constant thickness. We demonstrate that this phenomenon is ascribable to an increase in the interfacial dispersive forces that, enhancing physisorption of PET at the polymer/metal interface, causes a shortage of material at the crystal growth front.
\end{abstract}




\section{Introduction}

Polymer coatings of nanometric thickness are about to enter our everyday life in the form of protective layers, stimuli-responsive membranes, components of revolutionary flexible electronics devices, etc. ${ }^{1}$ With respect to thin films of inorganic materials, these "soft" systems are preferred because of easier and more versatile fabrication methods and the lack of degradation within long shelf time. ${ }^{2}$ Despite the tremendous literature available on this field, a full comprehension of the properties of these nanolayers has not been achieved yet. Thin polymer films, in fact, exhibit altered behavior with respect to the bulk, characterized by shifts in the phase transition temperatures and significant changes in other physical properties affecting processability, as flow, thermal expansion, mechanical stress, .... ${ }^{3-6}$

On this regard, similarly as for smaller molecules and liquid crystals, ${ }^{7}$ crystallization of polymers becomes slower upon confinement at the nanoscale level, ${ }^{8}$ where a severe reduction in the overall crystallization rate is commonly observed.9-11 In extreme cases, below a critical thickness (usually on the order of few tens of $\mathrm{nm}$ ) crystallization is not observed on time scales of industrial interest. ${ }^{12-13}$ The inhibition of crystallization upon confinement has attracted a considerable technological interest. Being able to suppress, or at least reduce, the crystallization rate would yield, for example, a tremendous increase in safety of those amorphous drugs where the crystalline form has non-negligible toxicity, ${ }^{14}$ and a neat improvement in the lifetime of polymer-based nanodevices, as coatings for optics and display applications where presence of crystals affects materials properties, ${ }^{8}$ e.g. opacity.

An operational parameter to evaluate the timescale of the formation of ordered structures is given by the crystallization time, $\mathrm{t}_{\mathrm{CRY}}$, a quantity, proportional to the inverse of the crystallization rate, that can be determined by monitoring the time evolution of the crystalline (or amorphous) volume fraction. In our previous work, we demonstrated that the thickness dependence of $t_{\mathrm{CRY}}$ can be written as the product of two terms, one related to the reduced 
nucleation probability and the other taking into account the lower diffusion rate in proximity of interfacial layers. ${ }^{13,15-17}$ To understand the physics behind the first term, we consider a polymer melt with homogenous nuclei density. In such case, a reduction of the thickness, $h$, achievable by considering thinner and thinner slabs of the above-mentioned melt, yields lower and lower probabilities to find active nuclei within a given portion of the slab. Such probability scales ${ }^{18}$ with $V^{-1}$, where $V$ is the sample volume. Working at constant sample surface, as in the case of most of the studies performed, results in a proportionality of the type $t_{C R Y} \sim h^{-1}$, which was verified by our previous experiments. ${ }^{13,15}$ The second term can be obtained by measurements of the segmental relaxation time $\tau$, which is related to diffusion $\left(\sim t_{C R Y}^{-1}\right)$ via a modified StokesEinstein relation, ${ }^{19} t_{C R Y} \sim \tau^{\xi}$ where $\xi$ is the Stokes-Einstein fractional exponent. This parameter typically assumes values between 1 and 0 , implying that crystallization (translational diffusion, mass transport ${ }^{16}$ ) becomes relatively faster than rotational dynamics as e.g. probed by measurements of glassy dynamics. ${ }^{20}$ As segmental dynamics can be affected by nanoconfinement ${ }^{4}$ a corresponding effect is expected also for diffusion and crystallization kinetics. Combining the contributions of nucleation and the diffusion, we got:

$$
t_{C R Y}(h, T)=\frac{\Lambda(T)}{h} \tau(h, T)^{\xi}
$$

where $\Lambda$ is a temperature dependent term related to bulk nucleation density and to the numerical value of $\xi$.

Eq 1 indicates that, for relatively thick films, the crystallization time increases upon confinement, with $h^{-1}$. A larger increment in $t_{C R Y}$ is expected for those systems, as poly(ethylene terephthalate) (PET) and poly(L-lactic acid) (PLLA), where segmental dynamics slows down upon confinement. Such contribution is observed in correspondence of the onset of slower interfacial dynamics, $h^{*}\left(\approx 20 \mathrm{~nm}^{21}\right)$.

Fitting experimental data of thin films of PLLA to Eq1, however, yields values of $\xi=2.7$, while for PET the increase in $t_{C R Y}$ with thickness for $h<20 \mathrm{~nm}$ is particularly sharp, hinting at values 
of $\xi \gg 1$, see Figure 1. Such exotic condition would correspond to a hindered mobility regime, where mass transport is less efficient than what predicted by the Stokes-Einstein relation in bulk. We hypothesized that this phenomenon could be due to the presence of adsorbed chains at the polymer/substrate interphase, whose conformations would inhibit translational diffusion. This argument is supported by several experimental observations. First, PET forms adsorbed layers thicker than PLLA, which is in line with the larger increase in $t_{C R Y}$ upon confinement of the former system. ${ }^{13,15}$ Second, experiments with chains of PEO show that, if a thick adsorbed layer is present, ${ }^{22-23}$ crystal growth is inhibited even in the presence of preexisting crystals.

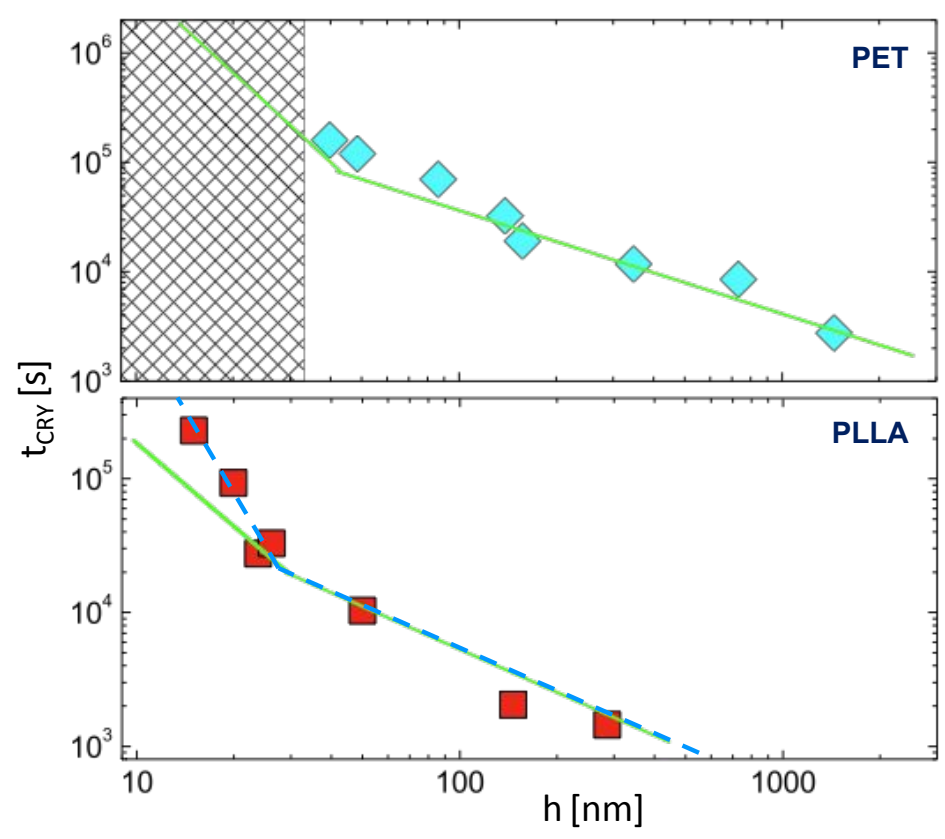

Figure 1 Thickness dependence of the crystallization time of thin films of poly(ethylene terephthalate) ${ }^{13}$ (top panel) and poly(L-lactic acid) ${ }^{15}$ (lower panel), in isothermal conditions. The shaded area indicates the thickness region where crystallization was not observed in PET up to 2 days of annealing at $373 \mathrm{~K}$. Fits of the experimental data to eq1 with $\xi=1$ (continuous green lines) and $\xi=2.7$ (dashed blue line) are also given.

With these ideas in mind, we hypothesized that it could be possible to control the crystallization kinetics of a sufficiently thin polymer film of constant thickness $\left(h<h^{*}\right)$ by varying the value of $\xi$, that is, by adequately processing the nanosystem to induce a different adsorption degree. 
A trivial way to achieve this goal would be holding the thin film for different annealing times above its glass transition temperature, $T_{g}$, to promote thickening of the interfacial adsorbed layer. However, because adsorption usually occurs at timescales much shorter than those of crystallization, ${ }^{13,15,22}$ such procedure cannot provide a precise control on the crystallization kinetics.

Based on recent work on polymer multilayers, ${ }^{24}$ we considered a different processing strategy. Here, we introduce a method permitting to tune crystallization rate of ultrathin polymer films without affecting film thickness or interfacial chemistry (same polymer layer / supporting substrate pair). This result is achieved by following recent work on the impact of nanoconfinement on interfacial interactions. ${ }^{24}$ As a proof of concept, we fabricated hybrid multilayers of the type $\mathrm{Al} / \mathrm{PET} / \mathrm{PS} / \mathrm{Al}$, and investigated the impact of a screening noncrystallizable layer of polystyrene (PS), separating PET from aluminum (Al) - adsorbing wall. Varying the thickness of the PS capping layer, we could adjust the amplitude of the dispersive forces acting at the interface between crystallizable layer and supporting substrate, and, hence, control the number of molecules irreversibly adsorbed. As the latter set of molecules do not take part to the crystallization process, we were able to tune the crystallization rate of the crystallizable layer, without altering its thickness.

\section{Materials and Methods}

Experiments Poly(ethylene terephthalate) $\left(\mathrm{M}_{\mathrm{n}}=38800 \mathrm{~g} \mathrm{~mol}{ }^{-1}, \mathrm{M}_{\mathrm{w}} / \mathrm{M}_{\mathrm{n}}=1.97\right)$ was dissolved in a trifluoroacetic acid/chloroform mixture and spincoated on top of an aluminum electrode, covered by a native layer of $\mathrm{Al}$ oxide $\left(\mathrm{h}_{\mathrm{AlOx}}=3 \mathrm{~nm}\right.$, as evaluated by ellipsometry), previously thermally evaporated onto clean glass plates. Solutions of polystyrene $\left(M_{w}=932 \mathrm{~kg} / \mathrm{mol}\right.$, $\mathrm{M}_{\mathrm{w}} / \mathrm{M}_{\mathrm{n}}=1.2$ ) in toluene (non-solvent for PET) were spincoated on top of the PET films. $4 \mathrm{~mm}^{2}$ nanocapacitors were finally obtained upon evaporation of a patterned top electrode. The 
thicknesses of single layers of PET $\left(14 \mathrm{~nm}<\mathrm{h}_{\mathrm{PET}}<1000 \mathrm{~nm}\right)$ and PS $\left(10 \mathrm{~nm}<\mathrm{h}_{\mathrm{PS}}<250 \mathrm{~nm}\right)$ were independently determined via ellipsometry and atomic force microscopy, further experimental details can be found in the Supporting Information. PS form sharp interfaces with PET (width $<1 \mathrm{~nm}$, even after prolonged annealing at $481 \mathrm{~K})^{25}$, which permits neglecting the effect of interdiffusion.

The complex dielectric permittivity, $\varepsilon^{*}=\varepsilon^{\prime}-i \varepsilon^{\prime \prime}$, was measured in isothermal conditions over a broad frequency range $(0.5 \mathrm{~Hz}-1 \mathrm{MHz}$ ) by continuous frequency sweeps (for $\mathrm{t}>50 \mathrm{~h}$ ) via a high-resolution dielectric analyzer. Thickness of the adsorbed layer was independently measured via atomic force microscopy and ellipsometry. To extract the thickness of the adsorbed layer, $\mathrm{h}_{\mathrm{ads}}{ }^{\mathrm{PET}}$, in the multilayer geometry, we annealed the sample at $373 \mathrm{~K}$ for $20 \mathrm{~min}$, a time larger than the characteristic adsorption time $(<1 \mathrm{~min})$, but smaller than $\mathrm{t}_{\mathrm{CRY}}$. Al was removed by pouring a few drops of a dilute solution of $\mathrm{NaOH}$ onto the upper metallic layer, the samples were then soaked in chloroform for $30 \mathrm{~min}$ to remove PS, and then in trifluoroacetic acid to wash out the unadsorbed PET chains. The adsorbed amount was obtained from $\mathrm{h}_{\mathrm{ads}}{ }^{\mathrm{PET}}$ via a standard procedure, described elsewhere. ${ }^{24,26}$

Determination of the crystallization time $t_{C R Y}$ Cold crystallization (devitrification of an amorphous system) was monitored via broadband dielectric spectroscopy (BDS) at $373 \mathrm{~K}$, where the dielectric response of PET is dominated by an intense peak in $\varepsilon$ " centered around fmax $\approx 10 \mathrm{kHz}$, commonly labeled as $\alpha$ - and attributed to the dynamic glass transition. The link between the intensity of the $\alpha$-peak, $\Delta \varepsilon$, that is the contribution of orientational polarization to the glassy dynamics, and the density of mobile dipole moments, allows following the crystallization kinetics via the conversion of the amorphous fraction. At $373 \mathrm{~K}$ the weaker dispersion of $\mathrm{PS}\left(\Delta \varepsilon_{\mathrm{PS}} / \Delta \varepsilon_{\mathrm{PET}}<0.07\right)$ is out of the experimentally accessible frequency window, $\log \left(\mathrm{f}_{\mathrm{max}} / \mathrm{f}_{\mathrm{PSax}}\right) \approx 7$, which permits a straightforward treatment of the experimental data. The 
neat response of PET was extracted from the total signal of the double layer (the two single layers were considered as capacitors in series ${ }^{27-28}$ ) and analyzed in terms of the empirical Havriliak-Negami function. ${ }^{29}$

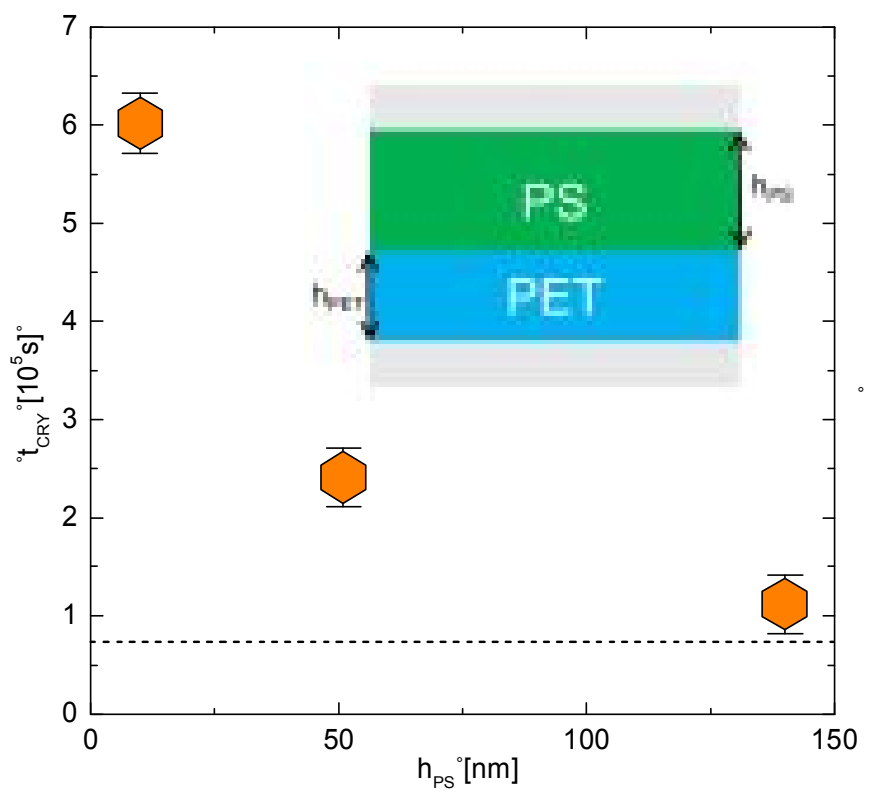

Figure 2 Isothermal crystallization time $(373 \mathrm{~K})$ for layers of $23 \mathrm{~nm}$ thick layers of PET embedded into two Al slabs, in parallel with layers of PS of different thickness, $h_{P S}$. The dashed line indicated the expected value of $\mathrm{t}_{\mathrm{CRY}}$ if only finite size effects were considered.

During annealing we observed a drop in the dielectric strength of the $\alpha$-peak, implying a reduction in the number of mobile segments. Prior to this phenomenon, a broader peak, indicated as $\alpha^{\prime}-$, emerged at lower frequencies. ${ }^{30}$ Ezquerra and coworkers demonstrated that the reduction in dielectric strength of the $\alpha$-peak does not follow the time evolution of the total crystalline content, because of the failure of a simple two-phase model in the presence of the complex molecular architecture of semi-crystalline polymers. ${ }^{31-32}$ However, simultaneous measurements of dielectric relaxation and X-ray scattering by Fukao et al verified that for PET the increase in crystalline volume fraction can be promptly monitored by following the drop in 
the sum of the intensities of the $\alpha$ - and the $\alpha^{\prime}$-processes (for sake of clarity, indicated here as the dielectric strength of PET).$^{30}$ In the case of thin films, we showed that irreversible chain adsorption is a concurrent source of immobilization and straightforwardly analyzed the reduction in dielectric strength as: ${ }^{13}$

$$
\Delta \varepsilon(t) / \Delta \varepsilon\left(t_{0}\right)=1-\chi\left[1-\exp \left(-t / t_{C R Y}\right)^{\beta}\right]-\delta \log \left(t / t_{0}\right)
$$

where $\chi$ indicates the drop in dielectric strength upon crystallization, $\delta$ is a parameter proportional to the amount of dipole moments immobilized upon adsorption, $\mathrm{t}_{0}$ indicates the onset of the measurement (set to $1 \mathrm{~s}$ for all samples) and $\beta$ is the Avrami exponent. ${ }^{33}$

Determination of the effective Hamaker constant We considered the multilayer system $\mathrm{Al}(1) / \mathrm{AlOx}(2) / \mathrm{PET}(3) / \mathrm{PS}(4) / \mathrm{Al}(5)$, where the numbers in parenthesis indicate the bottom-up order of the layers. While for sake of simplicity we will refer in the rest of the text as the PET/Al interface, here we explicitly took into account the presence of an oxide layerbetween $\mathrm{Al}$ and PET. The effective Hamaker constant ${ }^{34}$ of the forces acting at the PET/Al interface is given by

$$
A_{\text {eff }}=A_{234}-\frac{\sqrt{A_{121} A_{343}}}{\left(1+h_{A / O X} / h_{P E T}\right)^{3}}-\frac{\sqrt{A_{541} A_{323}}}{\left(1+h_{P S} / h_{P E T}\right)^{3}}+\frac{\sqrt{A_{545} A_{121}}}{\left(1+h_{A / O X} / h_{P E T}+h_{P S} / h_{P E T}\right)^{3}}
$$

where $\mathrm{A}_{\mathrm{XYZ}}$ is the nonretarded Hamaker constant for media $\mathrm{X}$ and $\mathrm{Z}$ interacting across medium $\mathrm{Y}$, that can be calculated ${ }^{34}$ from $\mathrm{A}_{\mathrm{XX}}, \mathrm{A}_{\mathrm{YY}}$ and $\mathrm{A}_{\mathrm{ZZ}}$ (with $\mathrm{A}_{\mathrm{jj}}$ the Hamaker constant of a system where two similar media act across a vacuum) as $A_{132} \approx\left(\sqrt{A_{11}}-\sqrt{A_{33}}\right)\left(\sqrt{A_{22}}-\sqrt{A_{33}}\right)$. We used $\mathrm{A}_{\mathrm{Al}}=40 \times 10^{-20} \mathrm{~J}^{34}, \mathrm{~A}_{\mathrm{Al} 2 \mathrm{O} 3}=16.75 \times 10^{-20} \mathrm{~J}^{35}, \mathrm{~A}_{\mathrm{PET}}=6.2 \times 10^{-20} \mathrm{~J}^{35}$ and $\mathrm{A}_{\mathrm{PS}}=7.9 \times 10^{-20} \mathrm{~J}^{34}$. Simavilla et $\mathrm{al}^{24}$ demonstrated that correction for retardation effects to eq 3 are not necessary in this kind of analysis.

\section{Results and discussion}


We prepared several multilayers Al/PET/PS/Al and investigated the crystallization kinetics at constant thickness of the PET layer, $\mathrm{h}_{\mathrm{PET}}$, while varying that of the PS layers, $\mathrm{h}_{\mathrm{PS}}$. Increasing $\mathrm{h}_{\mathrm{PS}}$ is expected to reduce the interfacial interactions between $\mathrm{Al}$ and $\mathrm{PET}^{24}$ and, hence, the number of PET chains adsorbed onto Al per unit surface, $\Gamma_{\mathrm{PET}}$. Based on our hypothesis, the latter phenomenon should speed up the crystallization kinetics, that is, an increase in $h_{P S}$ corresponds to a reduction in $\mathrm{t}_{\mathrm{CRY}}$.

Here, we first qualitatively present the results of our work and we later discuss them in term of the changes in dispersive forces in multilayers.

Results in Figure 2 show that we have been successfully able to tune the crystallization time, $t_{\mathrm{CRY}}$, of a 23-nm-thick film of PET by simply placing PS capping layers of different thickness. To understand the origin of this phenomenon, we considered experiments over a larger range of $h_{\text {PET }}$ and $h_{P S}$ values, see Figure 3 . Regardless of the thickness of the capping PS layers, when plotted as a function of the inverse thickness of the crystallizable layer $\mathrm{t}_{\mathrm{CRY}}$ shows two distinct regimes connected via a smooth transition.

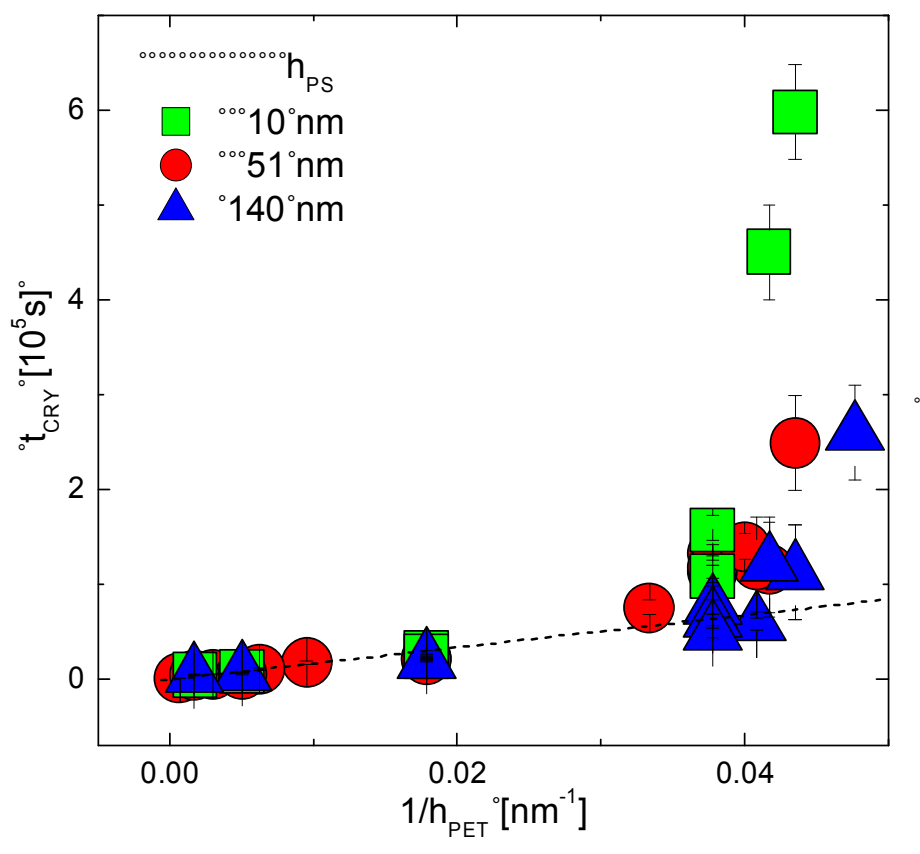


Figure 3 Isothermal crystallization time (373K) for layers of PET of thickness $h_{\text {PET }}$ embedded into two Al slabs, in parallel with layers of PS of thickness $h_{P S}$. The dashed line indicated the expected value of $t_{\mathrm{CRY}}$ if only finite size effects were considered.

Above thicknesses on the order of $\mathrm{h}_{\mathrm{PET}} * \approx 20-30 \mathrm{~nm}$, the changes in crystallization time are those typical of the nucleation limited regime, where only finite size effects are relevant $t_{\mathrm{CRY}}$ $\sim \mathrm{h}_{\mathrm{PET}}{ }^{-1} \cdot{ }^{13}$ In such regime, the thickness of the capping $\mathrm{PS}$ layer has no effect on the crystallization kinetics of PET.

For all the data series, regardless of the value of $h_{P S}$, a further reduction in thickness is accompanied by an increase in $\mathrm{t}_{\mathrm{CRY}}$, which, for $\mathrm{h}<\mathrm{h}_{\mathrm{PET}} *$, exceed the trend of thicker films based on nucleation effects. In this regime, at constant thickness of the crystallizable layer, an increase in $h_{\mathrm{PS}}$ corresponded to a reduction of $\mathrm{t}_{\mathrm{CRY}}$ capable to scale down, but not to suppress, the confinement effects.

This effect, not predicted by any current theory, is remarkable. The crystallization time of a $26.5 \mathrm{~nm}$ thick film of PET in the multilayer configuration, see Figure 2, is $16 \cdot 10^{3} \mathrm{~s}$ when $\mathrm{h}_{\mathrm{PS}}=$ $10 \mathrm{~nm}, 11 \cdot 10^{3} \mathrm{~s}$ for $\mathrm{h}_{\mathrm{PS}}=50 \mathrm{~nm}$ and drops to $5 \cdot 10^{3} \mathrm{~s}$ if $\mathrm{h}_{\mathrm{PS}}=250 \mathrm{~nm}$; for comparison, films of the same thickness in direct contact with two Al layers ${ }^{13}$ (a condition virtually occurring for $\mathrm{h}_{\mathrm{PS}}$ $\rightarrow 0$, if short-range forces are neglected) do not crystallize within $55 \mathrm{~h}$, that is $\mathrm{t}_{\text {cry }}>>200 \cdot 10^{3}$ $\mathrm{s}$, while much thicker films $\left(\mathrm{h}_{\mathrm{PET}}>>1 \mu \mathrm{m}\right)$ crystallize within $10 \mathrm{~min}$.

The experimental trends in Figures 1 and 2 do, hence, imply that the interfacial interactions between PET and Al decreases upon increase of the thickness of the PS layer. In such case, in fact, the number of chains of PET irreversibly adsorbed onto Al would decrease. 

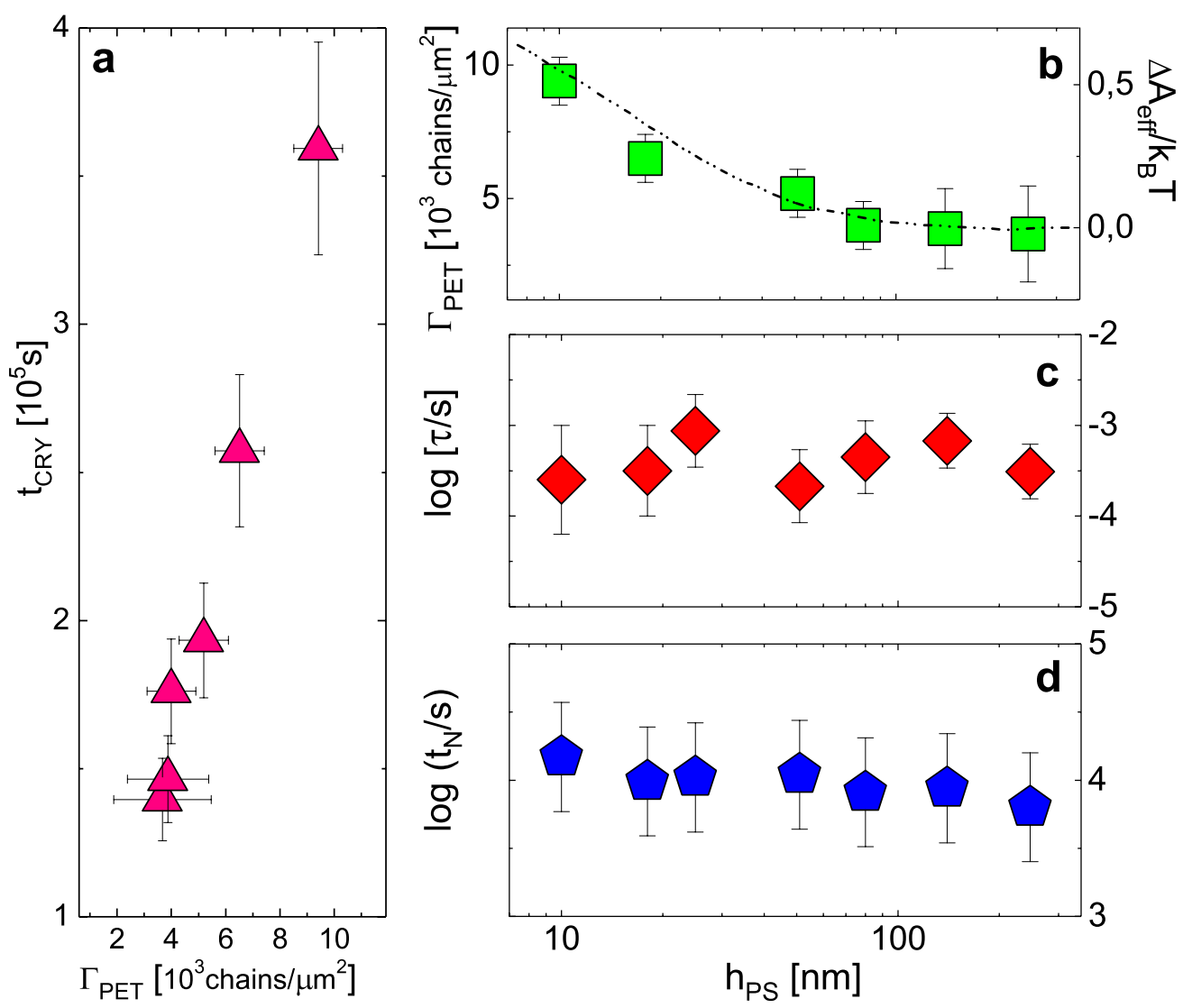

Figure 4 Properties of multilayers with constant $h_{\text {PET }}(26.5 \mathrm{~nm})$ and variable $h_{P S}$. a) Direct proportionality between the crystallization time and the thickness of the layer of PET irreversibly adsorbed on aluminum. b) Correlation between increase in Hamaker $(\Delta \mathrm{A})$ constant with respect to $250 \mathrm{~nm}$ thick PS films (dashed line) and the thickness of the layer of PET irreversibly adsorbed on aluminum (green circles); c) segmental relaxation time and d) nucleation time as a function of $h_{P S}$.

We tested whether our findings could be ascribed to the taming effect of interfacial interactions recently described by Simavilla et al., ${ }^{24}$ predicting a reduction in adsorbed PET amount upon increase of the thickness of the PS layer. The idea is based on the expected impact of $h_{P S}$ on the effective Hamaker constant, $\mathrm{A}_{\text {eff }},{ }^{34}$ of these hybrid system. The latter parameter quantifies the dispersion forces acting across PET between the two aluminum layers, originating from the interaction of fluctuating dipoles across different materials. Based on this reasoning, it is possible to correlate the value of the Hamaker constant with the depth of the intermolecular potential between PET and Al and, hence, to the number of chains adsorbed. 


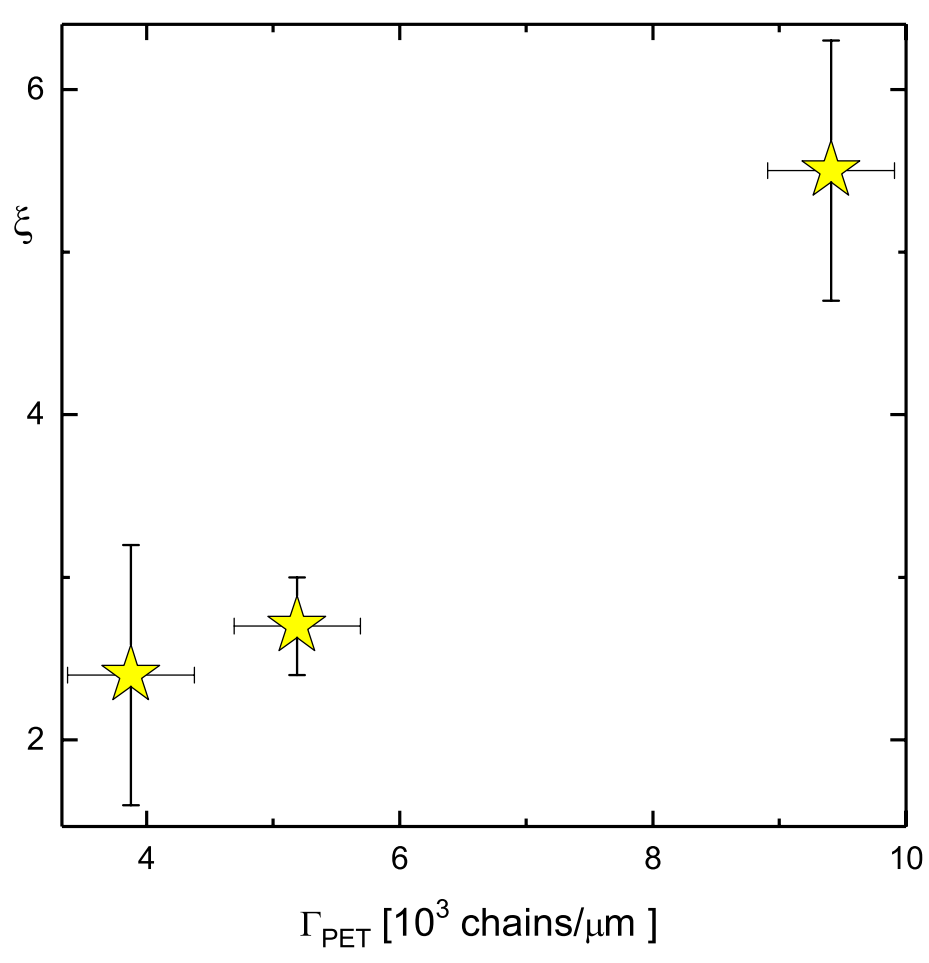

Figure 5 Proportionality between the adsorbed amount and the apparent Stokes-Einstein exponent of thin films of PET present in multilayers with constant $h_{\mathrm{PET}}(26.5 \mathrm{~nm})$ and variable $h_{\text {PS. }}$

In our multilayers, the effective Hamaker constant at the Al/PET interface is always negative, which implies that the multilayer are stable against desorption regardless of the thickness of the PS capping layer. $A_{\text {eff }}$ becomes less negative upon increase of $h_{P S}$, corresponding to smaller A1/PET interfacial interactions and thus smaller adsorbed amount. We experimentally validated this point by verifying that the number of PET chains adsorbed per unit surface, $\Gamma_{P E T}$, scales with $\mathrm{A}_{\text {eff, }}$, see Figure 4.b. In particular, we proved that at constant thickness of PET $\left(\mathrm{h}_{\mathrm{PET}}=26.5\right.$ $\mathrm{nm})$, a reduction of $\mathrm{h}_{\mathrm{PS}}$ from large values $(>100 \mathrm{~nm})$ to $15 \mathrm{~nm}$ provides an increment in longrange forces corresponding to an energy gain of $0.7 \mathrm{k}_{\mathrm{B}} \mathrm{T}$, sufficient to ensure irreversible chain adsorption. ${ }^{24}$ In particular the amount of PET chains adsorbed on Al increases by more than a factor 2 upon reduction of the PS capping layer. 
Based on Eq 1, the crystallization time is affected by three parameters, $\tau, \Lambda$ and $\xi$ respectively related to molecular mobility, nucleation rate and the decoupling between translational and rotational diffusion. In the following paragraphs we discuss on how $\mathrm{h}_{\mathrm{PS}}$ and interfacial forces could affect these three terms. In figure $4 \mathrm{c}$ we plotted the value of the segmental relaxation time of multilayers with constant $h_{\text {PET }}(26.5 \mathrm{~nm})$ and variable $h_{P S}$. No significant change is observed within experimental errors. This result was expected, because varying the thickness of the PS capping layer corresponds to a very small increase in the forces acting at the PET/Al interface, quantifiable as disjoining pressure would be the order of $10^{-4} \mathrm{~atm}$. Several orders of magnitude larger values (typically $0.1 \mathrm{GPa}$ ) are, instead, necessary to affect segmental dynamics. ${ }^{36-38}$ Similar arguments can be considered for the nucleation, because the variation in surface tension at the PET/PS interface is on the same order of magnitude of the disjoining pressure. ${ }^{39-40}$ These considerations are in agreement with the experimental results shown in figure $4 \mathrm{~d}$ where the nucleation time, $t_{N}$, of multilayers with constant $h_{P E T}(26.5 \mathrm{~nm})$, estimated as the onset of the changes in dielectric signal upon crystallization, ${ }^{41}$ is $\mathrm{h}_{\mathrm{PS}}$-invariant. This trend is also in line with the measured values of $t_{C R Y}$ in the nucleation-limited regime $\left(h_{\mathrm{PET}}>20-30 \mathrm{~nm}\right)$ where data from multilayers containing films of PS of different thickness do perfectly overlap.

Differently than for the two previous terms, the value of the Stokes-Einstein exponent, obtained by fitting the experimental data in Figure 3 to Eq 1, is significantly affected by the thickness of the capping layer. $\xi$ increases from 2.5 , a value comparable to that found for single layers of PLLA, see Figure 1, to 5.5 when $\mathrm{h}_{\mathrm{PS}}$ is reduced from 140 to $10 \mathrm{~nm}$. This trend implies that $\xi$ grows with $\Gamma_{\mathrm{PET}}$, see Figure 5, which verifies our hypothesis.

The perturbation in crystallization kinetics rate observed in our multilayers upon reduction of the noncrystallizable layer is, consequently, totally imputable to an increase in interfacial potential between crystallizable layer and the supporting Al interface which induces an increase in adsorbed amount. 
The increase in the number of adsorbed chains, at constant thickness of PET layer, implies a lower monomer density in the core of the film, that is, a shortage of crystallizable material. Consequently, at constant $h_{\mathrm{PET}}$, increasing $\mathrm{h}_{\mathrm{PS}}$ results in smaller neat driving forces for crystal growth, ${ }^{42}$ causing an increase in $\mathrm{t}_{\mathrm{CRY}}$. To further prove the validity of the hypothesis on the depletion of crystallizable material, we verified that the crystalline volume fraction reached at the end of the crystallization process $(\sim \chi$, see Eq 2$)$ increases with $h_{P S}$, see Figure 6 . Such results can be further parametrized by estimating the overall growth rate $G_{T O T}\left(\sim t_{C R Y}^{-1}\right)$ of a film of PET modeled as the sum of two chains populations, adsorbed vs nonadsorbed. Considering that the growth rate of the adsorbed chains is negligible ${ }^{13}$ with respect to that of the other molecules, G, we get $G_{T O T / \mathrm{G} \sim 1} 1-\Gamma_{\text {PET }} / m_{P E T}$, where $m_{P E T}\left(\sim h_{P E T}\right)$ is the total mass of the PET chains per unit area. In the case of experiments where the value of $h_{P E T}$ is kept constant, we find the scaling $t_{C R Y} \sim \Gamma_{P E T}$, which is verified by the data in Figure $4 \mathrm{a}$.

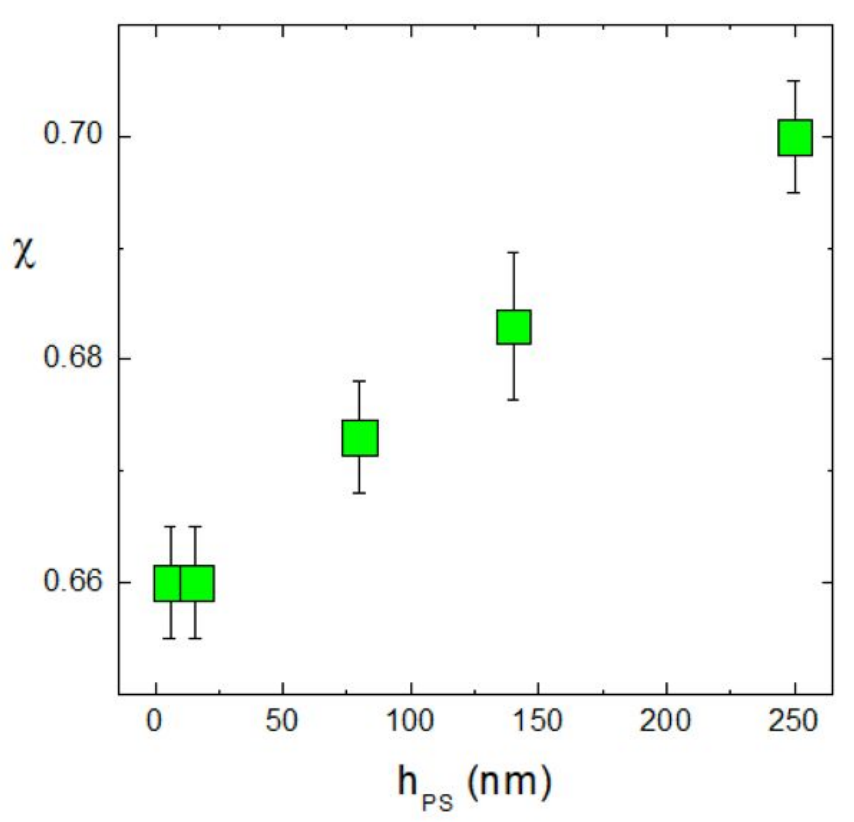

FIG 6 Impact of the thickness of PS on $\chi$ (see Eq 1), the amount of dipole moments immobilized at the end of the crystallization process for films of PET of constant thickness (26.5 nm). Larger $\chi$ values correspond to larger crystalline contents. We observe a reduction in $\chi$ upon reduction in $\mathrm{h}_{\mathrm{PS}}$, that is, for multilayers with more negative effective Hamaker constants. 
With these considerations in mind, we propose that, upon increase of the amount of chains adsorbed at the interfaces, the formation of ordered structures slows down due to the lesser availability of mobile chain at the growth front. During our experiments such a condition is always fulfilled, because the formation of an equilibrated adsorbed layer of PET onto Al is achieved before the onset of crystallization. ${ }^{13}$

Because $\Gamma_{P E T}$ smoothly increases at smaller $\mathrm{h}_{\mathrm{PS}}$, we expect that the shortage-induced reduction in growth rate would manifest around a critical thickness $h^{*}$ decreasing with the size of the screening layer, in line with the evidence in Figure 2. Finally, we remark that we could successfully explain the complex phenomenology affecting the crystallization of the PET layers by simply considering the changes in the number of PET chains irreversibly adsorbed on the Al substrate, and by neglecting any possible perturbation in the molecular conformations at the PET/PS interface. While we cannot exclude that a modification of the polymer/polymer interface could take place by varying $h_{\mathrm{PS}}$, the results of our experiments clearly indicate that the effect of such perturbations on the crystallization of the PET should be qualitatively similar to those described for the PET/Al interface or, eventually, negligible.

\section{Conclusion}

We verified that adequately capping a crystallizable film can be used to tune its crystallization kinetics. This process is mediated by a change in the amount of crystalline chains irreversibly adsorbed onto the supporting substrate, which induces a shortage of mobile chains at the crystal growth front. This phenomenon corresponds to a virtual increase in the Stokes-Einstein exponent, indicating less efficient mass transport. The method we introduced permitted us to achieve remote control of the crystallization kinetics of thin polymer films without changing the size of the crystallizable layer or the interfacial chemistry. Importantly, these effects are related to a perturbation in the amplitude of the interfacial interactions, that can be promptly 
determined before fabricating the multilayers, which ensures an efficient control of the processing conditions. ${ }^{43}$

Supporting information available Sample preparation and determination of the layer thicknesses, determination of the effective Hamaker constant, analysis of the crystallization kinetics, of the structural relaxation,

Acknowledgements B.V. and M.W. acknowledge financial support from the Research Council of the KU Leuven, Project No. OT/11/065, and financial support from FWO (Fonds Wetenschappelijk Onderzoeks-Vlaanderen), Project G.0642.08. S.N. acknowledge the Fonds de la Recherche Scientifique FNRS under Grant "EXOTICAGE" and ULB-ARC SADI

\section{References}

(1) Napolitano, S., Staying conductive in the stretch. Science 2017, 355, 24-25.

(2) Geoghegan, M.; Hadziioannou, G., Polymer Electronics. Oxford University Press: Oxford, 2013.

(3) Ediger, M. D.; Forrest, J. A., Dynamics near Free Surfaces and the Glass Transition in Thin Polymer Films: A View to the Future. Macromolecules 2014, 47, 471-478.

(4) Napolitano, S.; Glynos, E.; Tito, N. B., Glass transition of polymers in bulk, confined geometries, and near interfaces. Rep Prog Phys 2017, 80, 036602.

(5) Madkour, S.; Szymoniak, P.; Heidari, M.; von Klitzing, R.; Schonhals, A., Unveiling the Dynamics of Self-Assembled Layers of Thin Films of Poly(vinyl methyl ether) (PVME) by Nanosized Relaxation Spectroscopy. ACS Appl Mater Interfaces 2017, 9, 7535-7546. 
(6) Madkour, S.; Szymoniak, P.; Radnik, J.; Schonhals, A., Unraveling the Dynamics of Nanoscopically Confined PVME in Thin Films of a Miscible PVME/PS Blend. ACS Appl Mater Interfaces 2017, 9, 37289-37299.

(7) Grigoriadis, C.; Duran, H.; Steinhart, M.; Kappl, M.; Butt, H. J.; Floudas, G., Suppression of Phase Transitions in a Confined Rodlike Liquid Crystal. Acs Nano 2011, 5, 9208-9215.

(8) Liu, Y. X.; Chen, E. Q., Polymer crystallization of ultrathin films on solid substrates. Coordination Chemistry Reviews 2010, 254, 1011-1037.

(9) Despotopoulou, M. M.; Frank, C. W.; Miller, R. D.; Rabolt, J. F., Kinetics of chain organization in ultrathin poly(di-n-hexylsilane) films. Macromolecules 1996, 29, 5797-5804.

(10) Frank, C. W.; Rao, V.; Despotopoulou, M. M.; Pease, R. F. W.; Hinsberg, W. D.; Miller, R. D.; Rabolt, J. F., Structure in thin and ultrathin spin-cast polymer films. Science 1996, 273, 912-915.

(11) Massa, M. V.; Dalnoki-Veress, K.; Forrest, J. A., Crystallization kinetics and crystal morphology in thin poly(ethylene oxide) films. European Physical Journal E 2003, 11, 191198.

(12) Capitan, M. J.; Rueda, D. R.; Ezquerra, T. A., Inhibition of the crystallization in nanofilms of poly(3-hydroxybutyrate). Macromolecules 2004, 37, 5653-5659.

(13) Vanroy, B.; Wubbenhorst, M.; Napolitano, S., Crystallization of thin polymer layers confined between two adsorbing walls. Acs Macro Letters 2013, 2, 168-172.

(14) Beiner, M.; Rengarajan, G. T.; Pankaj, S.; Enke, D.; Steinhart, M., Manipulating the crystalline state of pharmaceuticals by nanoconfinement. Nano Letters $\mathbf{2 0 0 7 ,}$ 7, 1381 .

(15) Martinez-Tong, D. E.; Vanroy, B.; Wübbenhorst, M.; Nogales, A.; Napolitano, S., Crystallization of Poly(1-lactide) Confined in Ultrathin Films: Competition between Finite Size Effects and Irreversible Chain Adsorption. Macromolecules 2014, 47, 2354-2360. 
(16) Rodríguez-Tinoco, C.; Simavilla, D. N.; Priestley, R. D.; Wübbenhorst, M.; Napolitano, S., Density of Obstacles Affects Diffusion in Adsorbed Polymer Layers. ACS Macro Letters 2020, $9,318-322$.

(17) Napolitano, S.; Wübbenhorst, M., Anomalous decoupling of translational and rotational motion under 1D confinement, evidences from crystallization and diffusion experiments. In Dynamics in Geometrical Confinement, Springer: 2014; pp 279-306.

(18) Massa, M. V.; Dalnoki-Veress, K., Homogeneous crystallization of poly(ethylene oxide) confined to droplets: The dependence of the crystal nucleation rate on length scale and temperature. Physical Review Letters 2004, 92.

(19) Ngai, K.; Magill, J.; Plazek, D., Flow, diffusion and crystallization of supercooled liquids: Revisited. The Journal of Chemical Physics 2000, 112, 1887-1892.

(20) Mapes, M. K.; Swallen, S. F.; Ediger, M. D., Self-diffusion of supercooled o-terphenyl near the glass transition temperature. Journal of Physical Chemistry B 2006, 110, 507-511.

(21) Napolitano, S.; Capponi, S.; Vanroy, B., Glassy dynamics of soft matter under 1D confinement: how irreversible adsorption affects molecular packing, mobility gradients and orientational polarization in thin films. European Physical Journal E 2013, 36, 61.

(22) Jeong, H.; Napolitano, S.; Craig, B. A.; Priestley, R. D., Irreversible Adsorption Controls Crystallization in Vapor-Deposited Polymer Thin Films. J. Phys. Chem. Letters 2017, $8,229-234$.

(23) Asada, M.; Jiang, N.; Sendogdular, L.; Sokolov, J.; Endoh, M. K.; Koga, T.; Fukuto, M.; Yang, L.; Akgun, B.; Dimitriou, M.; Satija, S., Melt crystallization/dewetting of ultrathin PEO films via carbon dioxide annealing: the effects of polymer adsorbed layers. Soft Matter 2014, 10, 6392-403.

(24) Simavilla, D. N.; Huang, W.; Housmans, C.; Sferrazza, M.; Napolitano, S., Taming interfacial interactions via nanoconfinement. ACS Central Science 2018, 4 755-759. 
(25) Reiter, G.; Khanna, R., Negative excess interfacial entropy between free and end-grafted chemically identical polymers. Physical Review Letters 2000, 85, 5599-5602.

(26) Housmans, C.; Sferrazza, M.; Napolitano, S., Kinetics of Irreverisble Chain Adsorption. Macromolecules 2014, 47, 3390-3393.

(27) Peter, S.; Napolitano, S.; Meyer, H.; Wubbenhorst, M.; Baschnagel, J., Modeling Dielectric Relaxation in Polymer Glass Simulations: Dynamics in the Bulk and in Supported Polymer Films. Macromolecules 2008, 41, 7729-7743.

(28) Rotella, C.; Napolitano, S.; Wuebbenhorst, M., Segmental Mobility and Glass Transition Temperature of Freely Suspended Ultrathin Polymer Membranes. Macromolecules 2009, 42, 1415-1417.

(29) Havriliak, S.; Negami, S., A complex plane representation of dielectric and mechanical relaxation processes in some polymers. Polymer 1967, $8,161$.

(30) Fukao, K.; Miyamoto, Y., Dynamical transition and crystallization of polymers. Physical Review Letters 1997, 79, 4613-4616.

(31) Nogales, A.; Ezquerra, T. A.; Denchev, Z.; Sics, I.; Calleja, F. J. B.; Hsiao, B. S., Molecular dynamics and microstructure development during cold crystallization in poly(etherether-ketone) as revealed by real time dielectric and x-ray methods. Journal of Chemical Physics 2001, 115, 3804-3813.

(32) Ezquerra, T. A.; Sics, I.; Nogales, A.; Denchev, Z.; Balta-Calleja, F. J., Simultaneous crystalline-amorphous phase evolution during crystallization of polymer systems. Europhysics Letters 2002, 59, 417-422.

(33) Avrami, M., Kinetics of Phase Change. II Transformation - Time Relations for Random Distribution of Nuclei. Journal of Chemical Physics 1940, 8, 212.

(34) Israelachvili, J. N., Intermolecular and surface forces Academic Publisher: San Diego, 2011. 
(35) Lu, S.; Pugh, R. J.; Forssberg, E., Interfacial Separation of Particles Elsevier: Amsterdam, 2005.

(36) Paluch, M.; Pawlus, S.; Roland, C. M., Pressure and temperature dependence of the alpha-relaxation in poly(methyltolylsiloxane). Macromolecules 2002, 35, 7338-7342.

(37) Adrjanowicz, K.; Winkler, R.; Dziena, A.; Paluch, M.; Napolitano, S., Connecting 1D and 2D Confined Polymer Dynamics to Its Bulk Behavior via Density Scaling. Acs Macro Letters 2019, 8, 304-309.

(38) Papadopoulos, P.; Peristeraki, D.; Floudas, G.; Koutalas, G.; Hadjichristidis, N., Origin of the Glass Transition of Poly(2-vinylpyridine). A Temperature-and Pressure-Dependent Dielectric Spectroscopy Study. Macromolecules 2004, 37, 8116-8122.

(39) Müller, M.; MacDowell, G.; Müller-Buschbaum, P.; Wunnike, O.; Stamm, M., Nanodewetting: Interplay between van der Waals-and short-ranged interactions. Journal of Chemical Physics 2001, 115, 9960-9969.

(40) Macdowell, L. G.; Muller, M., Adsorption of polymers on a brush: Tuning the order of the wetting phase transition. J Chem Phys 2006, 124, 084907.

(41) Sanz, A.; Nogales, A.; Ezquerra, T. A., Influence of Fragility on Polymer Cold Crystallization. Macromolecules 2010, 43, 29-32.

(42) $\mathrm{Hu}, \mathrm{W}$., The melting point of chain polymers. The Journal of Chemical Physics 2000, $113,3901$.

(43) Chandran, S.; Baschnagel, J.; Cangialosi, D.; Fukao, K.; Glynos, E.; Janssen, L. M. C.; Mueller, M.; Muthukumar, M.; Steiner, U.; Xu, J.; Napolitano, S.; Reiter, G., Processing Pathways Decide Polymer Properties at the Molecular Level. Macromolecules 2019, 52, 71467156. 


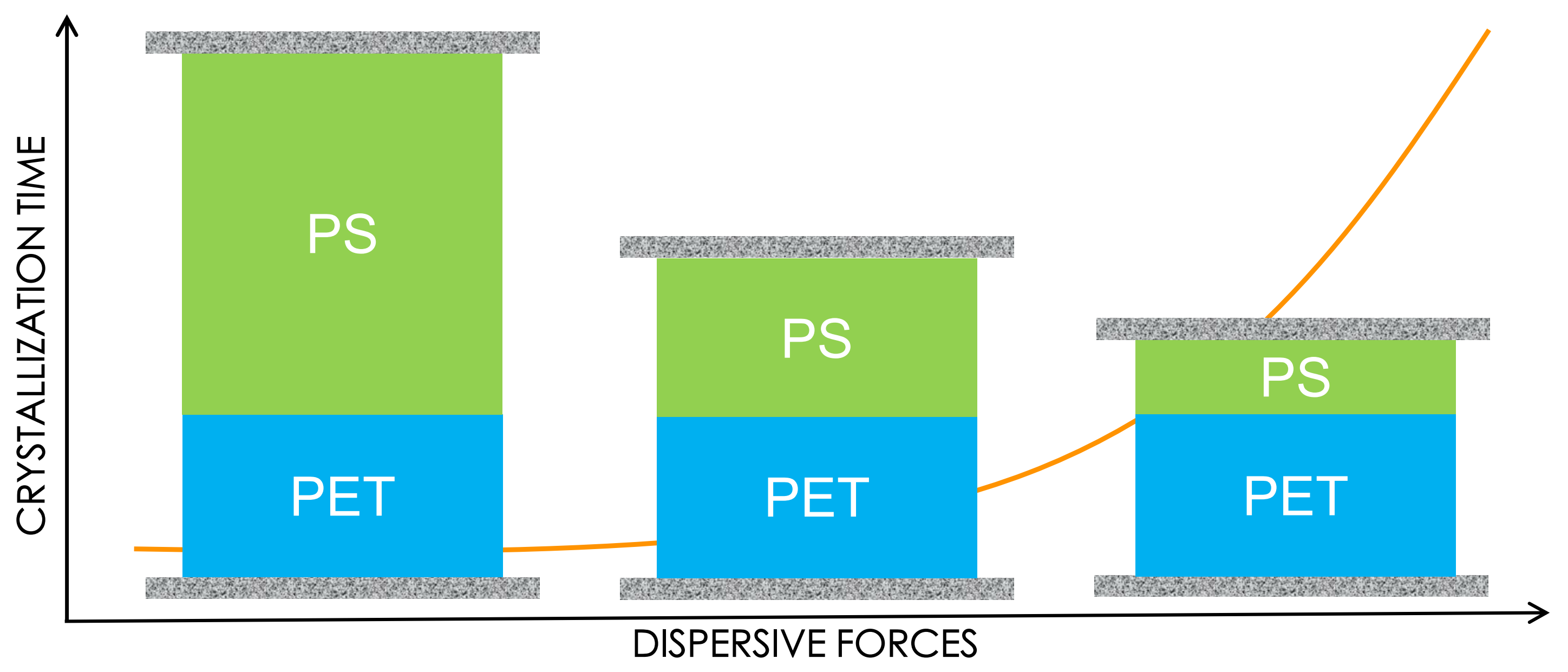

